PROCEEDINGS OF THE

AMERICAN MATHEMATICAL SOCIETY

Volume 128, Number 12, Pages 3471-3474

S $0002-9939(00) 05525-8$

Article electronically published on July 27, 2000

\title{
IRREDUCIBLE CONSTITUENTS OF FAITHFUL INDUCED CHARACTERS
}

\author{
I. M. ISAACS
}

(Communicated by Stephen D. Smith)

\begin{abstract}
Let $G$ be a finite group, and suppose $\chi$ is a character of $G$ obtained by inducing an irreducible character of some subgroup of $G$. If $\chi$ is faithful, we show that some irreducible constituent of $\chi$ has a solvable kernel. This yields an improved version of a theorem of Evdokimov and Ponomarenko.
\end{abstract}

\section{INTRODUCTION}

Suppose $G$ is a finite transitive permutation group with permutation character $\chi$, and let $d$ be the maximum of the degrees of the irreducible constituents of $\chi$. Recently, S. A. Evdokimov and I. N. Ponomarenko have shown that the (unique) largest solvable normal subgroup $\mathbf{S}(G)$ of $G$ must have index bounded above by some function of $d$. (See 22.) Specifically, they showed that $|G: \mathbf{S}(G)| \leq J(d)^{\log _{2}(d)}$, where $J(d)$ is the function associated with Jordan's theorem on finite complex linear groups. (See Theorem 14.12 of [4].) In other words, $J(d)$ is the smallest integer with the property that every finite subgroup $F$ of $G L(d, \mathbb{C})$ has an abelian normal subgroup $A$ such that $|F: A| \leq J(d)$.

One purpose of this paper is to give an easier proof of a more general result that yields a better bound.

Theorem A. Let $\psi \in \operatorname{Irr}(H)$, where $H \subseteq G$, and suppose that the induced character $\chi=\psi^{G}$ is faithful. If $d$ is the maximum of the degrees of the irreducible constituents of $\chi$, then $G$ has a solvable normal subgroup $S$ such that $|G: S| \leq J(d)$.

Note that if $\psi$ is the principal character of $H$, then $\chi$ is a faithful transitive permutation character, and we are in the situation considered by Evdokimov and Ponomarenko. We see, therefore, that Theorem A generalizes and strengthens the Evdokimov and Ponomarenko theorem, as promised

Theorem A is an easy corollary of the following result, which we consider to be the main theorem of this paper.

Theorem B. Let $\psi \in \operatorname{Irr}(H)$, where $H \subseteq G$, and suppose that the induced character $\chi=\psi^{G}$ is faithful. Then $\operatorname{ker}(\theta)$ is solvable for some irreducible constituent $\theta$ of $\chi$.

Received by the editors March 2, 1999.

2000 Mathematics Subject Classification. Primary $20 \mathrm{C} 15$.

This research was partially supported by the U.S. National Security Agency.

(C)2000 American Mathematical Society 
Proof of Theorem $A$. Let $\theta$ be an irreducible constituent of $\chi$ such that $K=\operatorname{ker}(\theta)$ is solvable. Then $G / K$ is isomorphic to a subgroup of $G L(n, \mathbb{C})$, where $n=\theta(1) \leq$ $d$, and hence $G / K$ is isomorphic to a subgroup of $G L(d, \mathbb{C})$. By Jordan's theorem, there exists an abelian subgroup $A / K \triangleleft G / K$ such that $|G: A|=|G / K: A / K| \leq$ $J(d)$. Of course, $A \triangleleft G$ and $A$ is solvable, and the proof is complete.

It is possible to improve Theorem B slightly, and to obtain an upper bound on the derived length of $\operatorname{ker}(\theta)$ in terms of the embedding of $H$ in $G$. This somewhat technical extension of Theorem B is the following.

Theorem C. In the situation of Theorem B, let $H=H_{0}<H_{1}<\cdots<H_{r}=G$ be a saturated chain of subgroups. (In other words, for each subscript $i$ with $0 \leq i<r$, assume that $H_{i}$ is maximal in $H_{i+1}$.) Then there exists an irreducible constituent $\theta$ of $\chi$ such that $\operatorname{ker}(\theta)$ is solvable of derived length at most equal to the number of indices $\left|H_{i+1}: H_{i}\right|$ that are prime powers.

As Evdokimov and Ponomarenko pointed out, an important special case of Theorem A is where $H=1$ and $\chi$ is the regular character of $G$. In that case, of course, every member of $\operatorname{Irr}(G)$ is a constituent of $\chi$, and thus $d=b(G)$, the maximum of the degrees of the irreducible characters of $G$. While Theorem A only guarantees the existence of a solvable normal subgroup of bounded index in $G$, an old theorem of D. S. Passman and the author [3] shows that $G$ actually has an abelian normal subgroup of bounded index. It is unclear, however, whether or not Theorem A can be improved to yield an abelian normal subgroup of bounded index in the general case. (As an alternative to [3], see Theorem 12.23 of [4] for a proof of the existence of a not necessarily normal abelian subgroup of index at most $(b(G) ! \cdot)^{2}$ This, of course, yields an normal abelian subgroup of index at most $\left((b(G) !)^{2}\right) !$.)

What happens in Theorem B if $\chi$ is the regular character? The conclusion is that for any finite group $G$, there exists $\theta \in \operatorname{Irr}(G)$ such that $\operatorname{ker}(\theta)$ is solvable. But a better result is known. In fact, there exists $\theta \in \operatorname{Irr}(G)$ such that $\operatorname{ker}(\theta)$ is nilpotent. (See [1] or Theorem 12.20 of [4] for this reeult of D. Broline.) We have been unable to decide, however, whether or not in the general situation of Theorem B, there must exist an irreducible constituent of $\chi$ having a nilpotent kernel.

\section{Proofs}

We begin with a lemma.

(2.1) Lemma. Let $M$ be a maximal subgroup of $G$, and suppose that $\alpha \in \operatorname{Irr}(M)$ is faithful, but that no irreducible constituent of $\alpha^{G}$ is faithful. Then $|G: M|$ is a prime power and the kernels of all irreducible constituents of $\alpha^{G}$ are abelian.

Proof. Let $\beta \in \operatorname{Irr}(G)$ be a constituent of $\alpha^{G}$. Write $K=\operatorname{ker}(\beta)$, and note that since $\alpha$ is a constituent of $\beta_{M}$, we have $K \cap M \subseteq \operatorname{ker}(\alpha)=1$. But $K>1$ by assumption, and thus $K \nsubseteq M$, and we conclude from the maximality of $M$ that $K M=G$. Also, since $K$ is a normal complement for the maximal subgroup $M$, it follows that $K$ is minimal normal in $G$, and thus $K$ is abelian if and only if it is a $p$-group for some prime $p$. Furthermore, $|K|=|G: M|$, and thus $K$ is abelian if and only if $|G: M|$ is a prime power.

Assuming, now, that $|G: M|$ is not a prime power, we work to derive a contradiction. Since $G=M K=M \operatorname{ker}(\beta)$, we see that $\beta_{M}$ is irreducible. Thus $\beta_{M}=\alpha$, and we have $\left[\alpha^{G}, \beta\right]=\left[\alpha, \beta_{M}\right]=1$. Since $\beta$ was an arbitrary irreducible constituent 
of $\alpha^{G}$, we see that $\alpha^{G}$ is a sum of distinct extensions of $\alpha$, and the number of these is $\alpha^{G}(1) / \alpha(1)=|G: M|$. Also, we know that the kernels of these extensions are normal complements for $M$ in $G$.

We claim that the kernels of the $|G: M|$ extensions of $\alpha$ to $G$ are distinct. To see why this is so, observe that, since $K M=G$, we have

$$
\left[\left(\alpha^{G}\right)_{K}, 1_{K}\right]=\left[\left(\alpha_{K \cap M}\right)^{K}, 1_{K}\right]=\left[\alpha_{K \cap M}, 1_{K \cap M}\right] \leq \alpha(1)=\beta(1)=\left[\beta_{K}, 1_{K}\right] .
$$

It follows that $K$ is not the kernel of any irreducible constituent of $\alpha^{G}$ other than $\beta$.

We now know that $M$ has $|G: M|$ distinct normal complements in $G$, and each of these is a nonabelian minimal normal subgroup. The product $U$ of these complements is therefore direct, and we see that $|U|=|G: M|^{|G: M|}$ and that $\mathbf{Z}(U)=1$. Also, we can write $U=K \times C$, where $K$ is a normal complement for $M$ and $C \triangleleft G$ with $|C|=|G: M|^{|G: M|-1}$. Because $|G: M|$ is not a prime power, we have $|G: M|>2$, and thus $|C|>|G: M|$. It follows that $C \cap M>1$.

Now $K$ normalizes $C \cap M$ since $K \subseteq \mathbf{C}_{G}(C)$. Also, $C \cap M \triangleleft M$ because $C \triangleleft G$, and it follows that $C \cap M \triangleleft K M=G$. But each of the normal complements to $M$ intersects $C \cap M$ trivially, and thus the normal subgroup $C \cap M$ centralizes each of them. It follows that $1<C \cap M \subseteq \mathbf{Z}(U)=1$, and this contradiction completes the proof.

Proofs of Theorems $B$ and $C$. If $H=G$, we see that $\psi=\chi$ is faithful, and thus $1=\operatorname{ker}(\psi)$ has derived length 0 , as desired. We can thus assume that $H<G$, and we work by induction on $|G: H|$. Let $H \subseteq M$, where $M$ is maximal in $G$, and let $r$ be the number of indices that are prime powers in a saturated chain of subgroups running from $H$ to $M$. Our task is to find an irreducible constituent $\theta$ of $\psi^{G}$ such that $\operatorname{ker}(\theta)$ is solvable of derived length at most $r$ if $|G: M|$ is not a prime power, and of derived length at most $r+1$ if $|G: M|$ is a prime power.

Let $\eta=\psi^{M}$, and write $N=\operatorname{ker}(\eta) \subseteq \operatorname{ker}(\psi) \subseteq H$. By the inductive hypothesis applied in the group $M / N$, we can choose an irreducible constituent $\alpha$ of $\eta$, with $\operatorname{ker}(\alpha)=L$, and such that $L / N$ is solvable with derived length at most $r$. Thus $L^{(r)} \subseteq N$, and if $K$ is a normal subgroup of $G$ contained in $L$, then $K^{(r)} \subseteq N \subseteq$ $\operatorname{ker}(\psi)$. Also $K^{(r)} \triangleleft G$, and it follows that $K^{(r)} \subseteq \operatorname{ker}\left(\psi^{G}\right)=\operatorname{ker}(\chi)=1$. In other words, every normal subgroup of $G$ contained in $L$ is solvable with derived length at most $r$.

Now let $\theta$ be any irreducible constituent of $\alpha^{G}$, and write $U=\operatorname{ker}(\theta) \triangleleft G$. Then $U \cap M \subseteq \operatorname{ker}\left(\theta_{M}\right) \subseteq \operatorname{ker}(\alpha)=L$. If $U \subseteq M$, therefore, we have $U \subseteq L$, and since $U \triangleleft G$, we see by the result of the previous paragraph that $U$ is solvable with derived length at most $r$. There is nothing further to prove in this case, and so we can assume $U \nsubseteq M$, and that this holds for every choice of the irreducible constituent $\theta$ of $\alpha^{G}$. In particular, since $U \triangleleft G$ and $M$ is maximal in $G$, we have $U M=G$. But $U=\operatorname{ker}(\theta)$, and it follows that $\theta_{M}$ is irreducible, and thus each of the irreducible constituents of $\alpha^{G}$ is an extension of $\alpha$. We can now apply Garrison's lemma, which is Lemma 12.17 of [4], and we deduce that $\mathbf{V}(\alpha) \triangleleft G$. (Recall that, by definition, $\mathbf{V}(\alpha)$ is the subgroup of $M$ generated by all elements $m \in M$ such that $\alpha(m) \neq 0$.) Now

$$
U \cap \mathbf{V}(\alpha) \subseteq U \cap M=\operatorname{ker}\left(\theta_{M}\right)=\operatorname{ker}(\alpha) \subseteq U \cap \mathbf{V}(\alpha)
$$

and so $L=\operatorname{ker}(\alpha)=U \cap \mathbf{V}(\alpha) \triangleleft G$. 
It follows that $L$ is solvable of derived length at most $r$, and that $\alpha$ is a faithful character of the maximal subgroup $M / L$ of $G / L$. We know that none of the irreducible constituents $\theta$ of $\alpha^{G}$ has kernel contained in $M$, and thus when viewed as a character of $G / L$, none of them is faithful. It follows by Lemma 2.1 that $|G: M|$ is a prime power and that $U / L$ is abelian. This shows that $\operatorname{ker}(\theta)=U$ is solvable of derived length at most $r+1$, and the proof is complete.

\section{REFERENCES}

[1] D. Broline, Nilpotence and character kernels, J. Algebra 45 (1977), 83-87. MR 55:473

[2] S. A. Evdokimov and I. N. Ponomarenko, Transitive groups with irreducible representations of bounded degree, Zap. Nauchn. Sem. S.-Peterburg. Otdel. Mat. Inst. Steklov. (POMI) 223 (1995), Teor. Predstav. Din. Sistemy, Kombin. i Algoritm. Metody. I, 108-119, 337-338. MR 97h:20004

[3] I. M. Isaacs and D. S. Passman, Groups with representations of bounded degree, Canad. J. Math. 161964 299-309. MR 29:4811

[4] I. M. Isaacs, Character Theory of Finite Groups, Dover, New York, 1994. CMP 94:14

Department of Mathematics, University of Wisconsin, 480 Lincoln Drive, Madison, WISCONSIN 53706

E-mail address: isaacs@math.wisc.edu 